\title{
Thermal Analysis of Selectively-Brominated Polystyrene
}

\author{
K. Pielichowski, ${ }^{\dagger}$ A. Puszyński, ${ }^{*}$ and J. Pielichowski \\ Institute of Organic Chemistry and Technology, Technical University of Kraków, \\ 31-155 Kraków, Warszawska 24, Poland \\ *Institute of Organic and Polymer Technology, Technical University of Wrociaw, \\ 50-370 Wrociaw, Wyspiańskiego 27, Poland
}

(Received October 21, 1993)

\begin{abstract}
In the present work thermogravimetric analysis (TGA) and differential thermal analysis (DTA) of selectively brominated polystyrene (PS) were carried out. The samples of PS were selectively brominated in the chain, on the ring, and also in both chain and ring. The effect of bromine content on the thermal stability was studied both in air and in inert atmosphere. Kinetic parameters of the degradation process (apparent activation energy, pre-exponential factor and order of reaction) were established using an efficient numerical procedure. Localization of bromine influenced the initial decomposition temperature (IDT) and the integral procedural decomposition temperature (IPDT). Samples containing bromine in the chain appeared to be the least stable. A possible explanation is given and mechanisms of degradation are suggested.

KEY WORDS Brominated Polystyrene / Thermal Analysis / Kinetics of Degradation /
\end{abstract}

Bromination of polystyrene (PS) is variously carried out to obtain graft polymers, ${ }^{1}$ highreactive modification and fire-resistant materials. $^{2}$ Depending on the reaction conditions bromination may lead to the introduction of $\mathrm{Br}$ solely into the ring or chain or into both areas of the PS structure. Hence, a free-radical bromination of PS in solution, in the presence of light and initiators, e.g., dibenzoyl peroxide or azobisisobutyronitrile, causes a Br substitution at the tertiary $\mathrm{C}$ atom in the polymer chain. ${ }^{3-6}$ The concentration of the initiator has neither influence on the content of bromine in PS, nor on its average molecular weight, but considerably affects the kinetics of bromination. ${ }^{6}$ Using $N$-bromocaprolactame as an bromination agent makes it possible to carry out the process without radical initiator, however the content of bromine remains low, reaching only $10 \% .^{6,7}$ Bromination of PS via an ionic route, conducted in solution in the

\footnotetext{
† To whom correspondence should be addressed.
}

presence of iron, aluminium chloride or iodine, leads to the introduction of $\mathrm{Br}$ into the aromatic ring. This reaction proceeds at room temperature in the absence of light. ${ }^{7,9,10}$ It has been shown that during a heterolytic bromination of PS in methylene chloride, at $30^{\circ} \mathrm{C}$ and in the presence of carbon tetrachloride, the bromination is $95 \%$ selective towards parasubstitution of the ring with respect to the polymer chain. ${ }^{11} \mathrm{~A}$ complex discussion on the bromination of atactic PS photoinitiated by $\mathrm{N}$-bromosuccinimide was conducted by Camps et al. ${ }^{12}$

It is well known that organic bromo compounds are effective flame retardants for polymeric materials. Since thermal decomposition is the first stage in the burning process, the thermal stability of the chemical species responsible for the flame retardation process obviously remains an important factor, which helps to improve the burning characteristics of 
a compound.

There appears to have been no recent work to describe the thermal stability of brominated PS in relation to the amount and localization of bromine in the polymer structure and to determine the kinetic parameters of the degradation process. Therefore, the present work attempts to evaluate the degradation behaviour of PS brominated in the chain, ring and also in both parts of polymer structure, using thermogravimetric analysis (TGA), its 1st derivative (DTG) and differential thermal analysis (DTA).

\section{EXPERIMENTAL PROCEDURES}

\section{Materials}

\section{PS Brominated in the Chain}

$13 \mathrm{~g}$ of PS (Aldrich, average molecular weight 90000) was dissolved in $90 \mathrm{~cm}^{3}$ of $\mathrm{CCl}_{4}$ and then brominated using $36 \mathrm{~g}$ of $\mathrm{N}$-bromosuccinimide in the presence of $3.6 \mathrm{~g}$ benzoyle peroxide for 2 hours at room temperature, then for the next 2 hours at $75^{\circ} \mathrm{C}$. After cooling the product was washed with a $2 \%$ solution of natrium pyrosulphite and reprecipitated by addition to methanol (20 times the reaction volume). The crude product was purified by redissolving it in benzene, reprecipitating and drying under vacuum.

One obtains $13.8 \mathrm{~g}$ of brominated PS (Brcontent $=21.0 \%$ ), corresponding to the introduction of $1 \mathrm{Br}$ atom per 2.9 PS repeat units. 2. PS Brominated on the Ring

$10.4 \mathrm{~g}$ of PS was dissolved in $100 \mathrm{~cm}^{3}$ of $\mathrm{CCl}_{4}$. Afterwards $0.2 \mathrm{~g}$ of pulverized iron was added, and, with a rigorous stirring, $5.1 \mathrm{~cm}^{3}$ of bromine was dropped in during 1 hour. The reaction was carried out at room temperature in the absence of light. After 24 hours a brown mixture was washed with a $2 \%$ solution of natrium pyrosulphite. The crude product was reprecipitated by addition to methanol, purified by redissolving in benzene and subsequently reprecipitated from methanol.

One obtains $10.8 \mathrm{~g}$ of the product with a
Table I. Characteristics of the samples used in the course of this work

\begin{tabular}{|c|c|c|c|c|}
\hline \multirow{2}{*}{$\begin{array}{c}\text { Number } \\
\text { of } \\
\text { sample }\end{array}$} & \multirow{2}{*}{$\frac{\mathrm{Br} \text { content }}{\mathrm{wt} \%}$} & \multicolumn{3}{|c|}{ Bromination } \\
\hline & & Chain & Ring & Both \\
\hline 1 & 0 & & & \\
\hline 2 & 3.0 & & $\mathrm{X}$ & \\
\hline 3 & 24.6 & $\mathrm{X}$ & & \\
\hline 4 & 29.9 & & & $\mathrm{X}$ \\
\hline 5 & 35.6 & & & $\mathrm{X}$ \\
\hline 6 & 45.8 & & $\mathrm{X}$ & \\
\hline 7 & 46.5 & & $\mathrm{X}$ & \\
\hline
\end{tabular}

bromine content of $45.1 \%$, corresponding to the introduction of $1 \mathrm{Br}$ atom per $0.93 \mathrm{PS}$ repeat units.

\section{PS Brominated in Both the Chain and on the Ring}

$7.2 \mathrm{~g}$ of PS brominated on the ring was dissolved in $90 \mathrm{~cm}^{3}$ of benzene and brominated using $15.4 \mathrm{~g}$ of $\mathrm{N}$-bromosuccinimide in the presence of $1.54 \mathrm{~g}$ of benzoyle peroxide at $80^{\circ} \mathrm{C}$ for 3 hours. After this time the mixture was shaken with a $2 \%$ solution of natrium pyrosulphite, then reprecipitated by addition to methanol.

One obtains $7.5 \mathrm{~g}$ of the product (Br-content $=33.7 \%$ ), corresponding to the introduction of $0.66 \mathrm{Br}$ atom per $1 \mathrm{PS}$ repeat unit.

A slight change in reaction conditions enabled us to obtain polymers with different bromine contents as shown in Table I.

\section{Thermal Analysis Techniques}

Thermogravimetric analysis (TGA) and differential thermal analysis (DTA) were performed using a Derivatograph-C thermal analyzer (MOM, Hungary). The conditions were: heating rate of $10 \mathrm{~K} \mathrm{~min}^{-1}$; sample mass of $10 \pm 1 \mathrm{mg}$; gas flow of $50 \mathrm{~cm}^{3} \mathrm{~min}^{-1}$ and $\mathrm{Al}_{2} \mathrm{O}_{3}$ as an inert material. The experiments have been carried out both in argon and air. Bromine content (in terms of wt $\%$ bromine) was estimated using the method of Schöniger. ${ }^{13}$ 


\section{RESULTS AND DISCUSSION}

The polymers under investigation were of the general formula

(i)

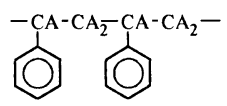

(ii)

(iii)
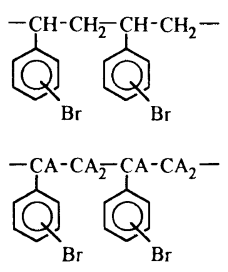

where $\mathrm{A}=\mathrm{H}$ or $\mathrm{Br}$

Localization of $\mathrm{Br}$ atoms in the chain was random and was not the subject of investigation.

Since the polymers contained bromine in different parts of the structure, it was expected that the interaction between these fragments and the main vinyl chain, and the electron system of the aromatic ring, would lead to differences in the thermal degradation process. In order to assess the influence of the gas atmosphere it is logical to show the results obtained in argon and in air separately.

\section{In Argon}

The results of thermogravimetric analysis (TGA), displayed in Table II, shows that samples 2, 6, and 7 (with bromine on the ring) undergo a degradation in higher temperatures than samples 3,4 , and 5, which posses $\mathrm{Br}$-atoms in the chain or in both chain and ring.

For the first group of samples the degradation process runs through one stage in a broad range of temperatures of $350-450^{\circ} \mathrm{C}$. Maxima of the decomposition rate, on the basis of $1 \mathrm{st}$ derivative of the weight loss curve, was found at $407-414^{\circ} \mathrm{C}$. Results of differential thermal analysis (DTA) reveal the occurrence of an endothermic heat effect at the conversion temperature. Samples 3, 4, and 5 undergo a gradual degradation already from $150^{\circ} \mathrm{C}$,
Table II. Temperatures $\left({ }^{\circ} \mathrm{C}\right)$ at which weight loss reached the levels specified on heating in argon measured by TGA

\begin{tabular}{lccccccc}
\hline & \multicolumn{7}{c}{ Weight loss } \\
\cline { 3 - 7 } Sample & \multicolumn{7}{c}{$\%$} \\
& \multicolumn{7}{c}{$\%$} \\
\cline { 2 - 7 } & 5 & 10 & 20 & 30 & 40 & 50 & 60 \\
\hline 1 & 370 & 378 & 385 & 390 & 395 & 400 & 405 \\
2 & 360 & 375 & 393 & 400 & 405 & 410 & 418 \\
3 & 238 & 288 & 340 & 368 & 387 & 412 & - \\
4 & 188 & 223 & 304 & 342 & 370 & 400 & 490 \\
5 & 218 & 250 & 334 & 360 & 382 & - & - \\
6 & 374 & 382 & 393 & 400 & 404 & 406 & 412 \\
7 & 380 & 387 & 394 & 401 & 404 & 407 & 413 \\
\hline
\end{tabular}

Table III. Temperatures $\left({ }^{\circ} \mathrm{C}\right)$ at which weight loss reached the levels specified on heating in air measured by TGA

\begin{tabular}{lccccccc}
\hline & \multicolumn{7}{c}{ Weight loss } \\
\cline { 3 - 7 } Sample & \multicolumn{7}{c}{$\%$} \\
& \multicolumn{7}{c}{$\%$} \\
\cline { 2 - 7 } & 5 & 10 & 20 & 30 & 40 & 50 & 60 \\
\hline 1 & 303 & 313 & 327 & 340 & 353 & 364 & 375 \\
2 & 355 & 372 & 381 & 386 & 392 & 400 & 404 \\
3 & 215 & 248 & 331 & 360 & 450 & - & - \\
4 & 187 & 221 & 298 & 339 & 385 & - & - \\
5 & 202 & 249 & 322 & 364 & 413 & - & - \\
6 & 329 & 348 & 360 & 371 & 378 & 398 & - \\
7 & 350 & 364 & 375 & 380 & 384 & - & - \\
\hline
\end{tabular}

having maxima of the rate of reaction in the range of $350-370^{\circ} \mathrm{C}$. The most thermally stable sample was the sample 7 , with an initial decomposition temperature (IDT) of $371^{\circ} \mathrm{C}$.

In Air

The thermogravimetric analysis in the atmosphere of air (Table III) shows that samples 2, 6, and 7 were more stable than samples 3,4 , and 5 , respectively.

Course of the degradation of all the samples from the first group remains similar until $400^{\circ} \mathrm{C}$. Above this temperature a mass decrease of $86 \%$ (at $500^{\circ} \mathrm{C}$ ) is detected for the sample 2. Other samples, except of sample 1, can be 
characterized by a char residue of $42-60 \%$. Samples 3, 4, and 5 undergo degradation in a two stage process: the first occurs up to $300^{\circ} \mathrm{C}$ and the second above this temperature. The maxima of the rate of reaction lie in the range of $345-360^{\circ} \mathrm{C}$.

From the comparison of thermal analysis results obtained in oxidative (air) and inert gas (argon) atmosphere can be observed, as expected, that degradation occurs at lower temperatures for the measurements in air. This is particularly distinct for sample 1 (PS). Simultaneously, the char residue of sample 7 at $500^{\circ} \mathrm{C}$ was about $43 \%$ more in air than in argon, suggesting that decomposition products created in an oxidative atmosphere are more thermally stable in this temperature range.

To find the values of the kinetic parameters (apparent activation energy, pre-exponential factor and order of reaction) of the degradation process a special integral procedure was used. ${ }^{14}$ Assuming, as a kinetic decomposition model, an $n$-th order irreversible reaction, in form of

$$
\frac{\mathrm{d} \alpha}{\mathrm{d} t}=k(1-\alpha)^{n}
$$

where $\alpha$, the degree of conversion; $t$, time; $k$, the rate constant; $n$, the order of reaction

leads, after some rearrangements and integration, to the following relationship

$$
\frac{A}{\beta} \int \exp \left(\frac{-E}{R T}\right) \mathrm{d} T=\int \frac{\mathrm{d} \alpha}{(1-\alpha)^{n}}
$$

where $A$, the pre-exponential factor; $R$, the gas constant; $T$, the absolute temperature; $E$ the apparent activation energy; $\beta$, the heating rate

The results of the kinetic investigations are collected in Table IV. It can be seen that values of activation energy are lower for the samples containing bromine in the chain or in both the chain and ring than for the samples possessing bromine exclusively on the ring.

Analysis of the influence of the $\mathrm{Br}$ content on the thermal stability described by IDT
Table IV. The estimated values of the kinetic parameters of the degradation process in argon atmosphere

\begin{tabular}{crcc}
\hline & \multicolumn{1}{c}{$E$} & \multicolumn{1}{c}{$A$} \\
\cline { 2 - 3 } Sample & $\mathrm{kJ} \mathrm{mol}^{-1}$ & $\mathrm{~s}^{-1}$ & $n$ \\
\hline 1 & 143.8 & $3.06 \times 10^{6}$ & 0.38 \\
2 & 129.2 & $3.52 \times 10^{6}$ & 0.31 \\
$3^{*}$ & 59.5 & $6.49 \times 10^{4}$ & 2.19 \\
$4^{*}$ & 57.4 & $4.92 \times 10^{3}$ & 2.18 \\
$5^{*}$ & 58.9 & $5.08 \times 10^{3}$ & 1.93 \\
6 & 169.7 & $2.93 \times 10^{12}$ & 0.17 \\
7 & 183.7 & $3.60 \times 10^{13}$ & 0.27 \\
\hline
\end{tabular}

* For $\alpha \in[0.2 ; 0.5]$.

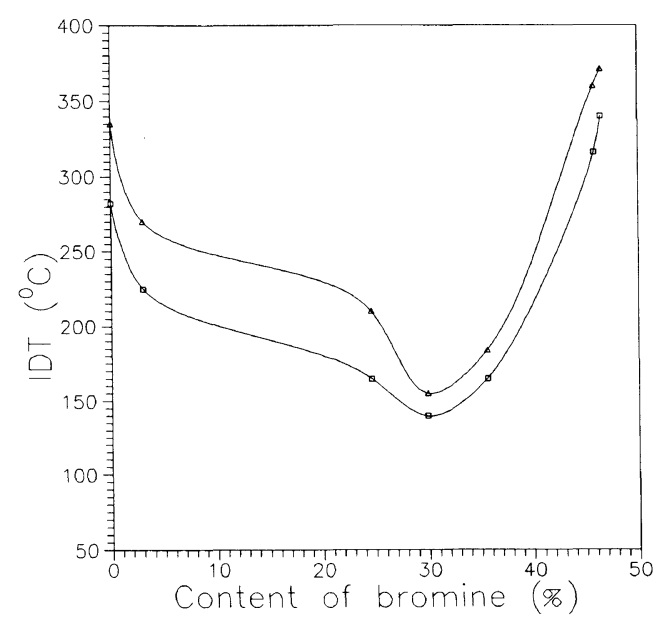

Figure 1. Variation of IDT with respect to bromine content in PS in argon $(\triangle)$ and air $(\square)$ atmospheres.

values shows that samples 6 and 7 containing the highest global amount of bromine posses the highest IDT values (Figure 1).

On the other hand the lowest values of IDT correspond to samples which have a $\mathrm{Br}$ content in the middle range, $24.6-35.6 \%$, placed both in the chain and on the ring. Values of the integral procedural decomposition temperature (IPDT), evaluated according to Doyle, ${ }^{15}$ are higher in argon than in air. Degradation is more intensive in the presence of oxygen thus decreasing IPDT values. They are the lowest for samples 3, 4, and 5, analogous as in case 


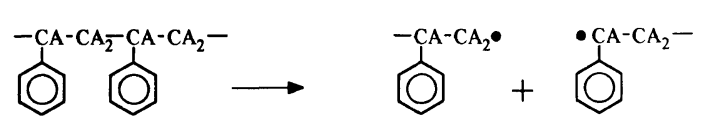

Scheme 1.

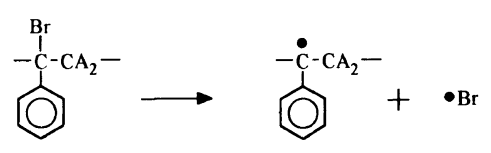

Scheme 2.

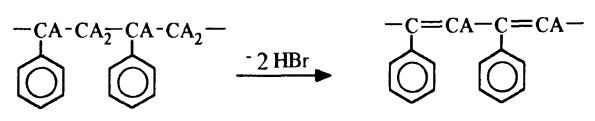

Scheme 3.

of IDT. It seems that the location of $\mathrm{Br}$ atoms in the polymer structure plays a key role. Hence, for the structure (i) activation energies of dissociation of $\mathrm{C}-\mathrm{Br}$ bonds as proposed in Scheme 1 lie in the range $45-70 \mathrm{kcal} \mathrm{mol}^{-1} \cdot{ }^{16}$ When bromine is bonded to the benzene ring much higher energy is required to brake down this structure. From the three types of $\mathrm{C}-\mathrm{Br}$ bonds, i.e., $-\mathrm{CH}_{2}-\mathrm{Br},=\mathrm{CH}-\mathrm{Br}$, and $\equiv \mathrm{C}-\mathrm{Be}$, the stability of the latter one is the lowest and hence the decomposition starts at the tertiary $\mathrm{C}$ atom. Therefore for the degradation of the structure (i) the following reaction can be suggested as initial (Scheme 2). On the other hand, comparing with the PVC degradation ${ }^{17}$ and according to the results of thermal volatilisation analysis (TVA), ${ }^{18}$ formation of $\mathrm{HBr}$ would lead to the polyene sequences, displayed in Scheme 3. This would considerably affect the mass loss, being responsible for the first degradation stage, as reported for polyvinyl bromide. ${ }^{19}$ However, this phenomenum requires detailed analysis.

The thermogravimetric curves, describing mass loss vs. temperature, show global mass effects of partial reactions (connected with mass change), contributing to the whole mechanism. Introducing bromine into the chain strongly influences the thermal behavior of the polymer, whose stability depends now

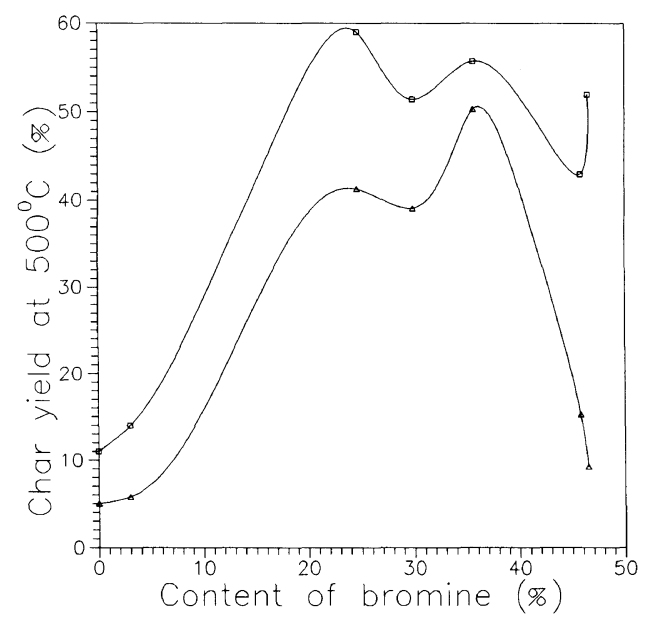

Figure 2. Variation of char yield at $500^{\circ}$ with respect to bromine content in PS in argon $(\triangle)$ and air $(\square)$ atmospheres.

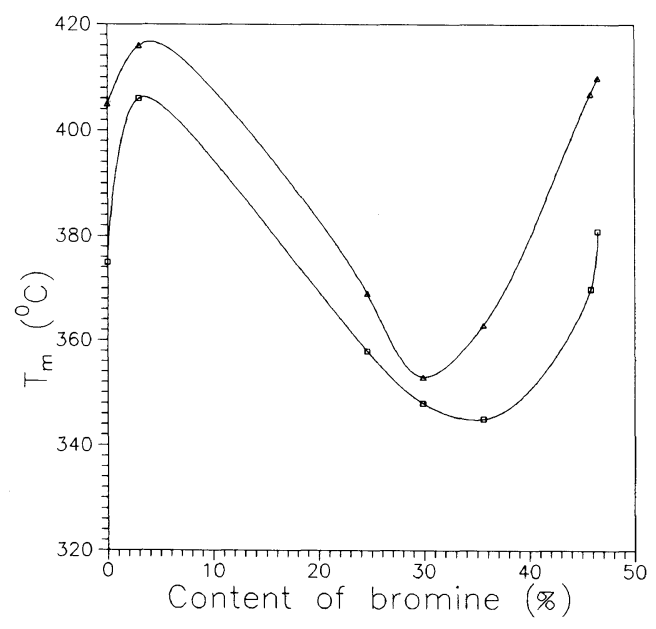

Figure 3. Variation of temperature of maximum degradation rate $\left(T_{\mathrm{m}}\right)$ with respect to bromine content in PS in argon $(\triangle)$ and air $(\square)$ atmospheres.

on the strength of $\mathrm{C}-\mathrm{Br}$ bonds. Br-containing structures then play an active role in the process of degradation, leading to an increased formation of non-volatile products. The mechanism is still a subject of debate. ${ }^{20-23}$ It is worth mentioning, according to the plot " $\mathrm{Br}$ content vs. char yield" displayed in Figure 2, that samples 3, 4, and 5, with $\mathrm{Br}$ contents of $24.6-35.6 \%$, yield the highest amount of char 
in the atmosphere of air. In this case intermediate products are probably more stable than those of the other samples, and can be formed at lower temperatures, since temperatures corresponding to the maxima of decomposition rate differ considerably (Figure 3).

Summarizing the results it can be indicated that samples containing bromine in the chain were less thermally stable than these which possessed bromine on the ring. Localization of bromine in the polymer structure considerably affected values of IPD, IPDT, and char residue. In order to better understand the degradation mechanism, it may be necessary to determine precisely the degradation products. Such studies are now in progress.

Acknowledgments. The authors are grateful Dr. Ian Hamerton from the University of Surrey, England, for his advice during preparation of this paper.

\section{REFERENCES}

1. F. Candan and P. R. Remp, Makromol. Chem., 122, 15 (1969).

2. P. Hodge anỏ D. C. Sherington, "Polymer Supported Reactions in Organic Syntheses,” Wiley Interscience,
New York, N.Y.; 1980.

3. D. Braun, Kunststoffe, 50, 375 (1960).

4. P. Teyssic, M. C. de Wilde, and G. Smets, J. Polym. Sci., 16, 429 (1955).

5. M. M. Jones, Canadian J. Chem., 34, 948 (1956).

6. I. M. Panaitow, Makromol. Chem., 52, 70 (1962).

7. G. Maura and F. Pochetti, Ann. Chim., 58, 40 (1960).

8. A. E. Qureshi and W. T. Ford, Reactive Polym., 10, 279 (1989).

9. G. B. Bachmann, J. Org. Chem., 12, 108 (1947).

10. S. N. Ushakov and P. A. Matuzov, Angew. Chem., 17, 538 (1944).

11. M. Camps, J. P. Montheard, F. Benrokia, J. M. Camps, and Q. T. Pham, Eur. Polym. J., 26, 53 (1990).

12. M. Camps, A. Jebri, P. Verlaque, A. Archavlis, and R. Faure, Eur. Polym. J., 29, 99 (1993).

13. W. Schöniger, Mikrochim. Acta, 74 (1954).

14. J. Pielichowski, S. Kedzierski, and K. Pielichowski, submitted to Journal of Thermal Analysis.

15. C. D. Doyle, Anal. Chem., 33, 77 (1961).

16. T. Kelen, "Polymer Degradation," Van Nostrand Reinhold, New York, N.Y., 1983.

17. E. D. Owen, Ed., "Degradation and Stabilisation of PVC," Elsevier Applied Science Publishers, London and New York, 1984.

18. I. C. McNeill and M. Coskun, Polymer Degradation Stability, 25, 1 (1989).

19. I. C. McNeill, T. Straiton, and P. Anderson, J. Polym. Sci., Polym. Chem. Ed., 18, 2085 (1980).

20. H. H. G. Jellinek, Ed., "Degradation and Stabilization of Polymers," Vol. 1, Elsevier, New York, N.Y., 1983.

21. C. Bouster, J. Anal. Appl. Pyrol., 15, 249 (1989).

22. L. Dean, Polym. Degrad. Stabil., 25, 143 (1989).

23. J. Kristina, Eur. Polym. J., 25, 767 (1989). 